\title{
ABSTRACT SEMILINEAR STOCHASTIC ITÓ-VOLTERRA INTEGRODIFFERENTIAL EQUATIONS
}

\author{
DAVID N. KECK AND MARK A. MCKIBBEN
}

Received 31 October 2005; Revised 3 March 2006; Accepted 14 April 2006

We consider a class of abstract semilinear stochastic Volterra integrodifferential equations in a real separable Hilbert space. The global existence and uniqueness of a mild solution, as well as a perturbation result, are established under the so-called Caratheodory growth conditions on the nonlinearities. An approximation result is then established, followed by an analogous result concerning a so-called McKean-Vlasov integrodifferential equation, and then a brief commentary on the extension of the main results to the time-dependent case. The paper ends with a discussion of some concrete examples to illustrate the abstract theory.

Copyright (c) 2006 D. N. Keck and M. A. McKibben. This is an open access article distributed under the Creative Commons Attribution License, which permits unrestricted use, distribution, and reproduction in any medium, provided the original work is properly cited.

\section{Introduction}

Let $(\Omega, \mathfrak{F}, P)$ be a complete probability space equipped with a filtration $\left\{\mathfrak{F}_{t}: t \geq 0\right\}$. We investigate a class of abstract stochastic integrodifferential equations of the form

$$
\begin{gathered}
d x(t ; \omega)=L(x(t ; \omega)) d t+f(t, x(t ; \omega) ; \omega) d t \\
+g(t, x(t ; \omega) ; \omega) d W(t), \quad 0 \leq t \leq T \\
x(0 ; \omega)=x_{0}(\omega),
\end{gathered}
$$

in a separable Hilbert space $H$, where the operator $L$ is one of the following forms:

$$
\begin{gathered}
L(x(t ; \omega))=\int_{0}^{t} a(t-s ; \omega) A x(s ; \omega) d s, \\
L(x(t ; \omega))=A\left[x(t ; \omega)+\int_{0}^{t} a(t-s ; \omega) x(s ; \omega) d s\right] .
\end{gathered}
$$

Hindawi Publishing Corporation

Journal of Applied Mathematics and Stochastic Analysis

Volume 2006, Article ID 45253, Pages 1-22

DOI 10.1155/JAMSA/2006/45253 
Here, $A: D(A) \subset H \rightarrow H$ is a linear, closed, densely-defined (possibly unbounded) operator; $x:[0, T] \times \Omega \rightarrow H, f:[0, T] \times H \times \Omega \rightarrow H$, and $g:[0, T] \times H \times \Omega \rightarrow \mathfrak{L}^{2}(K ; H)$ (where $K$ is another real separable Hilbert space and $\mathfrak{L}^{2}(K ; H)$ denotes the space of all HilbertSchmidt operators from $K$ into $H$ ) are given mappings; and $a:[0, T] \times \Omega \rightarrow \mathbb{R}$ is a stochastic kernel. Also, $W$ is a $K$-valued cylindrical Wiener process and $x_{0}$ is an $\mathfrak{F}_{0}$-measurable $H$-valued random variable independent of $W$. Hereafter, for brevity, we suppress the dependence on $\omega \in \Omega$ in our notation unless needed.

Deterministic integrodifferential equations of the form

$$
\begin{gathered}
x^{\prime}(t)+L(x(t))=f(t, x(t)), \quad 0 \leq t \leq T, \\
x(0)=x_{0}
\end{gathered}
$$

have been extensively considered by Prüss $[28,29]$ and others (see $[2-6,8,13,14]$ ) for

$$
L(x(t))=\int_{0}^{t} a(t-s) A x(s) d s .
$$

Also, (1.4) with

$$
L(x(t))=A\left[x(t)+\int_{0}^{t} a(t-s) x(s) d s\right]
$$

has been studied in [21-23], for both the case when $A$ is autonomous and when $A$ is timedependent. For $L$ of either form above, under appropriate conditions such as [29, Theorem 1.4, page 46], (1.4) admits a resolvent family $\{R(t): t \geq 0\}$ in the following sense.

Definition 1.1. A family $\{R(t): t \geq 0\}$ of bounded linear operators on $H$ is a resolvent for (1.4) whenever

(i) $R(t)$ is strongly continuous in $t$,

(ii) $R(0)=I$,

(iii) $R(t) D(A) \subset D(A)$ and $A R(t) z=R(t) A z$, for all $z \in D(A), t \geq 0$,

(iv) $(d R(t) / d t) z=z+(a * A R) z=z+(R * A a) z$,

where $*$ denotes the usual convolution over $[0, t]$. (See [29, page 32].)

Assuming the classical Lipschitz condition on $f$, it has been shown (see $[21,29])$ that there exists a unique mild solution on $[0, T]$, for any $T>0$, that can be represented by the variation of parameters formula involving the resolvent family, namely,

$$
x(t)=R(t) x_{0}+\int_{0}^{t} R(t-s) f(s, x(s)) d s, \quad 0 \leq t \leq T .
$$

Certain applications, such as those mentioned in [21-26], indicate that a stochastic version of (1.4) warrants study. Indeed, Mīshura [24-26] studied the stochastic Volterra integral equation

$$
x(t ; \omega)=L(x(t ; \omega))+f(t ; \omega), \quad 0 \leq t \leq T,
$$

(with $L$ given by (1.2)) and established conditions under which such an equation could be reduced to one involving Skorokhod integrals (to allow, in particular, for a natural 
treatment of the equations used to describe the motion of an incompressible viscoelastic fluid). As pointed out in that paper, the results mentioned above in the deterministic case can be applied for each given fixed $\omega \in \Omega$ to ensure the existence of a resolvent family $\{R(t ; \omega): t \geq 0\}$. However, the stochastic version must be $\mathfrak{F}_{t}$-adapted, and so, to guarantee this, certain natural conditions (such as those in [25, Theorem 2]) are imposed; these conditions hold for a broad class of operators.

The purpose of the present investigation is to continue the above work by considering the more general Itó-Volterra integrodifferential equation (1.1) which contains an additional stochastic term involving a Wiener process. The main results in the present paper constitute an extension of the results in $[13,21-23]$ to the stochastic setting, and can be viewed as a counterpart to the results in $[24,26]$ under more general growth conditions. Moreover, we consider a so-called McKean-Vlasov variant of (1.1) in which the mappings $f$ and $g$ depend on the probability law $\mu(t)$ of the state process $x(t)$ (i.e., $\mu(t) B=P(\{\omega \in \Omega: x(t ; \omega) \in B\})$ for each Borel set $B$ on $H)$. Precisely, we study

$$
\begin{gathered}
d x(t ; \omega)=L(x(t ; \omega)) d t+f(t, x(t ; \omega), \mu(t ; \omega) ; \omega) d t \\
+g(t, x(t ; \omega), \mu(t ; \omega) ; \omega) d W(t), \quad 0 \leq t \leq T, \\
x(0 ; \omega)=x_{0}(\omega) .
\end{gathered}
$$

A prototypical example of such a problem in the finite-dimensional setting would be an interacting $N$-particle system in which (1.9) describes the dynamics of the particles $x_{1}, \ldots, x_{N}$ moving in the space $H$ in which the probability measure $\mu$ is taken to be the empirical measure $\mu_{N}(t)=(1 / N) \sum_{k=1}^{N} \delta_{x_{k}(t)}$, where $\delta_{x_{k}(t)}$ denotes the Dirac measure. Researchers have investigated related models concerning diffusion processes in the finitedimensional case (e.g., see $[10,11,27]$ ) and have more recently devoted attention to the study of the infinite-dimensional version (see $[1,19])$. Our discussion of (1.9) serves as a counterpart to these results for a class of stochastic Volterra equations.

We will be concerned with mild solutions to (1.1) in the following sense.

Definition 1.2. (i) An $H$-valued stochastic process $\{x(t): 0 \leq t \leq T\}$ is a mild solution of (1.1) (with $L$ given by (1.2)) if

(a) $x(t)$ is $\mathfrak{F}_{t}$-adapted,

(b) $x \in \mathscr{C}([0, T] ; H)$,

(c) $b * x \in \mathscr{C}\left([0, T] ;\left(D(A),\|\cdot\|_{A}\right)\right)$, where $b(t)=\int_{0}^{t} a(s) d s$,

(d) $x(t)=x_{0}+A(b * x)(t)+\int_{0}^{t} f(s, x(s)) d s+\int_{0}^{t} g(s, x(s)) d W(s)$.

(ii) An $H$-valued stochastic process $\{x(t): 0 \leq t \leq T\}$ is a mild solution of (1.1) (with $L$ given by (1.3)) if it satisfies (i) with (c) and (d) replaced by

(c') $x$ and $a * x \in \mathscr{C}\left([0, T] ;\left(D(A),\|\cdot\|_{A}\right)\right)$,

$\left(\mathrm{d}^{\prime}\right) x(t)=x_{0}+\int_{0}^{t}(A x)(s) d s+\int_{0}^{t}(a * A x)(s) d s+\int_{0}^{t} f(s, x(s)) d s+\int_{0}^{t} g(s, x(s)) d W(s)$.

In the case when (1.1) admits a resolvent family, a mild solution in both cases of Definition 1.2 can be represented by a stochastic version of (1.7), namely,

$$
x(t)=R(t) x_{0}+\int_{0}^{t} R(t-s) f(s, x(s)) d s+\int_{0}^{t} R(t-s) g(s, x(s)) d W(s), \quad 0 \leq t \leq T .
$$


The structure of this paper is as follows. In Section 2 we state some preliminary information regarding function spaces and inequalities. Then, we state the main results concerning existence and uniqueness of mild solutions of (1.1), along with an approximation result, a discussion of a so-called McKean-Vlasov variant of (1.1), and commentary on analogous results for the time-dependent case in Section 3. We provide the proofs in Section 4, and finally present a discussion of some examples in Section 5.

\section{Preliminaries}

For details of this section and additional background, we refer the reader to $[9,14,17$, $18,29]$ and the references therein. Throughout this paper, $H$ and $K$ are real separable Hilbert spaces with respective norms $\|\cdot\|_{H}$ and $\|\cdot\|_{K}$. Several function spaces are used throughout the paper. As mentioned earlier, $\mathfrak{L}^{2}(K ; H)$ denotes the space of all HilbertSchmidt operators from $K$ into $H$ with norm denoted as $\|\cdot\|_{\mathfrak{L}^{2}(K ; H)}$. The space of all bounded linear operators on $H$ will be denoted by $B(H)$ with norm $\|\cdot\|_{B(H)}$, while the collection of all strongly measurable square integrable $H$-valued random variables $x$ is denoted by $L^{2}(\Omega ; H)$ equipped with norm

$$
\|x(\cdot)\|_{L^{2}(H)}=\left(E\|x(\cdot ; \omega)\|_{H}^{2}\right)^{1 / 2} .
$$

For any Banach space $Z, \mathscr{C}([0, T] ; Z)$ stands for the function space

$$
\left\{v \in \mathscr{C}\left([0, T] ; L^{2}(\Omega ; Z)\right): v(t) \text { is } \mathfrak{F}_{t} \text {-adapted, } 0 \leq t \leq T\right\}
$$

which is itself a Banach space when equipped with the norm

$$
\|v\|_{\mathscr{C}(Z)}=\sup _{0 \leq t \leq T}\left(E\|v(t ; \cdot)\|_{Z}^{2}\right)^{1 / 2}
$$

and $L^{p}(0, T ; Z)$ represents the space

$$
\left\{v \in L^{p}\left([0, T] ; L^{2}(\Omega ; Z)\right): v(t) \text { is } \mathfrak{F}_{t} \text {-adapted, } 0 \leq t \leq T\right\}
$$

with the usual norm. We abbreviate these two spaces as $\mathscr{C}(Z)$ and $\mathscr{L}^{p}(Z)$, respectively.

When considering (1.9), we will make use of the following additional function spaces used in [1]. First, $\mathfrak{B}(H)$ stands for the Borel class on $H$ and $\mathfrak{P}(H)$ represents the space of all probability measures defined on $\mathfrak{B}(H)$ equipped with the weak convergence topology. Let $\lambda(x)=1+\|x\|_{H}, x \in H$, and define the space

$$
\mathfrak{C}_{\rho}(H)=\left\{\varphi: H \longrightarrow \mathbb{R} \mid \varphi \text { is continuous and }\|\varphi\|_{\mathfrak{C}_{\rho}}<\infty\right\}
$$

where

$$
\|\varphi\|_{\mathfrak{C}_{\rho}}=\sup _{x \in H} \frac{|\varphi(x)|}{\lambda^{2}(x)}+\sup _{x \neq \bar{x} \text { in } H} \frac{|\varphi(x)-\varphi(\bar{x})|}{\|x-\bar{x}\|_{H}}<\infty .
$$


Let $m=m^{+}-m^{-}$be the Jordan decomposition of $m,|m|=m^{+}+m^{-}$, and for $p \geq 1$, let

$$
\begin{gathered}
\mathfrak{P}_{\lambda^{p}}^{s}(H)=\{m: \mathfrak{B}(H) \longrightarrow \mathbb{R} \mid m \text { is a signed measure on } H \text { such that } \\
\left.\|m\|_{\lambda^{p}}=\int_{H} \lambda^{p}(x)|m|(d x)<\infty\right\} .
\end{gathered}
$$

Then, we can define the space $\mathfrak{P}_{\lambda^{2}}(H)=\mathfrak{P}_{\lambda^{2}}^{s}(H) \cap \mathfrak{P}(H)$ equipped with the metric $\rho$ given by

$$
\rho\left(\nu_{1}, \nu_{2}\right)=\sup \left\{\left\langle\varphi, \nu_{1}-\nu_{2}\right\rangle \equiv \int_{H} \varphi(x)\left(\nu_{1}-\nu_{2}\right)(d x):\|\varphi\|_{\mathfrak{C}_{\rho}} \leq 1\right\}
$$

it has been shown that $\left(\mathfrak{P}_{\lambda^{2}}(H), \rho\right)$ is a complete metric space. Finally, the space of all continuous $\mathfrak{P}_{\lambda^{2}}(H)$-valued measures defined on $[0, T]$, denoted by $\mathscr{C}_{\lambda^{2}}(T)$, is complete when equipped with the metric

$$
D_{T}\left(\nu_{1}, \nu_{2}\right)=\sup _{t \in[0, T]} \rho\left(\nu_{1}(t), \nu_{2}(t)\right), \quad v_{1}, \nu_{2} \in \mathscr{C}_{\lambda^{2}}(T) .
$$

The following estimate on the Itó integral established in [16, Proposition 1.9] is an important tool in obtaining certain estimates.

LEMmA 2.1. If $Y:[0, T] \times \Omega \rightarrow \mathfrak{L}^{2}(K ; H)$ is a strongly measurable random variable with $\int_{0}^{T} E\|Y(t)\|_{\mathfrak{L}^{2}(K ; H)}^{p} d t<\infty$, for a given $p \geq 2$, then there exists a positive constant $L_{Y}$ such that

$$
E\left\|\int_{0}^{t} Y(s) d W(s)\right\|_{H}^{p} \leq L_{Y} t^{(p-2) / 2} \int_{0}^{t} E\|Y(s)\|_{\mathfrak{L}^{2}(K ; H)}^{p} d s .
$$

Finally, in addition to the familiar Young, Hölder, and Minkowski inequalities, the following inequality (which follows from the convexity of $x^{m}, m \geq 1$ ) plays an important role in establishing various estimates,

$$
\left(\sum_{i=1}^{n} a_{i}\right)^{m} \leq n^{m-1} \sum_{i=1}^{n} a_{i}^{m}
$$

where $a_{i}$ is a nonnegative constant $(i=1, \ldots, m)$.

\section{Statement of main results}

We begin by establishing the existence and uniqueness of a mild solution to (1.1) (in the sense of Definition 1.2) under the so-called Caratheodory growth conditions (see [12]). Precisely, we consider (1.1) in a real separable Hilbert space $H$ under the following conditions:

(H1) $A: D(A) \subset H \rightarrow H$ is a closed, linear densely-defined (possibly unbounded) operator;

(H2) $a(t)$ is $\mathfrak{F}_{t}$-adapted with sample paths $a(\cdot ; \omega)$ such that $\int_{0}^{T}|a(s ; \omega)| d s<\infty$ a.s.;

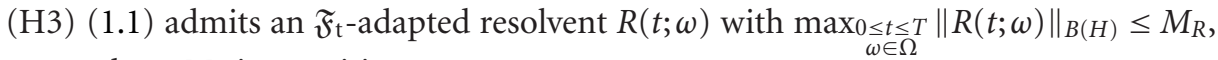
where $M_{R}$ is a positive constant; 
(H4) $f:[0, T] \times H \rightarrow H, g:[0, T] \times H \rightarrow \mathfrak{L}^{2}(K ; H)$ are $\mathfrak{F}_{\mathrm{t}}$-measurable mappings satisfying

(i) there exists $K:[0, \infty) \times[0, \infty) \rightarrow[0, \infty)$ such that

(a) $K(\cdot, \cdot)$ is continuous in both variables, and nondecreasing and concave in the second variable,

(b) $E\|f(t, x)\|_{H}^{2}+E\|g(t, x)\|_{\mathfrak{L}^{2}(K ; H)}^{2} \leq K\left(t, E\|x\|_{H}^{2}\right)$, for all $0 \leq t \leq T$ and $x \in L^{2}(\Omega ; H)$

(ii) there exists $N:[0, \infty) \times[0, \infty) \rightarrow[0, \infty)$ such that

(a) $N(\cdot, \cdot)$ is continuous in both variables, and nondecreasing and concave in the second variable with $N(t, 0)=0$,

(b) $E\|f(t, x)-f(t, y)\|_{H}^{2}+E\|g(t, x)-g(t, y)\|_{\mathfrak{L}^{2}(K ; H)}^{2} \leq N\left(t, E\|x-y\|_{H}^{2}\right)$, for all $0 \leq t \leq T$ and $x, y \in L^{2}(\Omega ; H)$;

(H5) the function $N$ of (H4)(ii) is such that if a nonnegative continuous function $z:[0, T] \rightarrow[0, \infty)$ satisfies $z(0)=0$ and

$$
z(t) \leq D \int_{0}^{t} N(s, z(s)) d s, \quad \forall 0 \leq t \leq T,
$$

for an appropriate positive constant $D$, then $z(t)=0$, for all $0 \leq t \leq T$;

(H6) for any fixed $T>0, \beta>0$, the initial-value problem

$$
u^{\prime}(t)=\beta K(t, u(t)), \quad u(0)=u_{0} \geq 0
$$

has a global solution on $[0, T]$;

(H7) $x_{0}$ is an $\mathfrak{F}_{0}$-measurable random variable in $L^{2}(\Omega ; H)$ independent of $W$.

Examples of functions Nsatisfying (H4)(ii) and (H5) can be found in [12, 15]. Aside from the mapping that would generate a Lipschitz condition (namely $N(t, u)=M u$, for some positive constant $M$ ), some other typical examples (see [15]) for the mapping $N$ in (H4)-(H5) include

$$
\begin{gathered}
N(t, \cdot)=t \ln \left(\frac{1}{t}\right), \quad t \in\left(0, t_{0}\right], \\
N(t, \cdot)=t \ln \left(\frac{1}{t}\right) \ln \left(\ln \left(\frac{1}{t}\right)\right), \quad t \in\left(0, t_{0}\right] .
\end{gathered}
$$

Conditions that ensure (H3) holds are discussed, for instance, in [25].

We have the following theorem.

Theorem 3.1. If (H1)-(H7) hold, then (1.1) has a unique mild solution $x \in \mathscr{C}([0, T] ; H)$.

Furthermore, we assert that uniqueness is guaranteed to be preserved under sufficiently small perturbations. Indeed, consider a perturbation of (1.1) given by

$$
\begin{gathered}
d x(t)=L(x(t)) d t+[f(t, x(t))+\tilde{f}(t, x(t))] d t \\
+[g(t, x(t))+\tilde{g}(t, x(t))] d W(t), \quad 0 \leq t \leq T, \\
x(0)=x_{0} .
\end{gathered}
$$

An argument in the spirit of [7] can be used to establish the following result. 
Proposition 3.2. Assume that (H1)-(H7) hold, and that $\tilde{f}$ and $\tilde{g}$ satisfy (H4) and (H5) with appropriate mappings $\tilde{K}$ and $\tilde{N}$. Then, (3.4) has a unique mild solution, provided that (H8) there exist $\delta \in(0, T)$ and $w \in C((0, \infty) ;(0, \infty))$ which is nondecreasing and $\int_{1}^{\infty}(d u / w(u))=\infty$ such that $\tilde{N}(r, \cdot) \leq N(r, \cdot) w\left(\int_{r}^{1}(d u / N(u))\right)$, for all $r \in(0, \delta)$.

In the case of a Lipschitz growth condition, routine calculations can be used to establish the following estimates.

Proposition 3.3. Assume that (H1)-(H7) hold (with $N(t, u)=K(t, u)=M u$, for some $M>0$ ) and that $x_{0}, \tilde{x}_{0}$ satisfy (H7). Denote the corresponding unique mild solutions of (1.1) (as guaranteed to exist by Theorem 3.1 ) respectively by $x, \tilde{x}$. Then,

(i) there exist $\beta_{1}, \beta_{2}>0$ such that

$$
E\|x(t)-\tilde{x}(t)\|_{H}^{2} \leq \beta_{1}\left(1+\left\|x_{0}-\tilde{x}_{0}\right\|_{L^{2}(H)}^{2}\right) \exp \left(\beta_{2} t\right), \quad \forall 0 \leq t \leq T,
$$

(ii) for each $p \geq 2$, there exists a positive constant $C_{p, T}$ (depending only on $p$ and $T$ ) such that

$$
\sup _{t \in[0, T]} E\|x(t)\|_{H}^{2 p} \leq C_{p, T}\left(1+E\left\|x_{0}\right\|_{H}^{p}\right)
$$

We now formulate a result in which a related deterministic Volterra integrodifferential equation (as considered in $[21,29]$ ) is approximated by a sequence of stochastic equations of the form (1.1). Precisely, consider the deterministic initial-value problem

$$
\begin{gathered}
z^{\prime}(t)+L(z(t))=f(t, z(t)), \quad 0 \leq t \leq T, \\
z(0)=x_{0} .
\end{gathered}
$$

For every $\varepsilon>0$, consider the stochastic initial-value problem

$$
\begin{gathered}
d x_{\varepsilon}(t)=L_{\varepsilon}\left(x_{\varepsilon}(t)\right) d t+f_{\varepsilon}\left(t, x_{\varepsilon}(t)\right) d t+g_{\varepsilon}\left(t, x_{\varepsilon}(t)\right) d W(t), \quad 0 \leq t \leq T, \\
x_{\varepsilon}(0)=x_{0} .
\end{gathered}
$$

Here, $L$ and $L_{\varepsilon}$ are given by either (1.2) or (1.3). Also, assume that $L, f$, and $g$ satisfy (H1)-(H4) (appropriately modified) with $N(t, u)=K(t, u)=M u$, for some $M>0$ (i.e., $f$ and $g$ satisfy a Lipschitz condition) so that the results in $[21,29]$ guarantee the existence of a unique global mild solution $z$ of (3.7). Regarding (3.8), we impose the following conditions, for every $\varepsilon>0$ :

(H9) $A_{\varepsilon}: D\left(A_{\varepsilon}\right)=D(A) \subset H \rightarrow H$ and $a_{\varepsilon}$ satisfy (H1)-(H2). Also, (3.8) admits an $\mathfrak{F}_{t^{-}}$ adapted resolvent $\left\{R_{\varepsilon}(t): t \geq 0\right\}$ such that $R_{\varepsilon}(t) \rightarrow R(t)$ strongly as $\varepsilon \rightarrow 0^{+}$, uniformly in $t \in[0, T]$ and $\left\{R_{\varepsilon}(t): 0 \leq t \leq T\right\}$ is uniformly bounded by $M_{R}$ (the same constant as defined in (H3), independent of $\varepsilon$ );

(H10) $f_{\varepsilon}:[0, T] \times H \rightarrow H$ is Lipschitz in the second variable (with the same Lipschitz constant $M$ as for $f$ and $g$ ) and $f_{\varepsilon}(t, z) \rightarrow f(t, z)$ as $\varepsilon \rightarrow 0^{+}$, for all $z \in H$, uniformly in $t \in[0, T]$;

(H11) $g_{\varepsilon}:[0, T] \times H \rightarrow \mathfrak{L}^{2}(K ; H)$ is Lipschitz in the second variable (with the same Lipschitz constant $M$ as for $f$ and $g$ ) and $g_{\varepsilon}(t, z) \rightarrow 0$ as $\varepsilon \rightarrow 0^{+}$, for all $z \in H$, uniformly in $t \in[0, T]$. 
Under these assumptions, Theorem 3.1 ensures the existence of a unique mild solution of (3.8), for every $\varepsilon>0$. We have the following convergence result.

Theorem 3.4. Let $z$ and $x_{\varepsilon}$ be the mild solutions to (3.7) and (3.8), respectively. Then, there exist $\xi>0$ and a positive function $\Psi(\varepsilon)$ which decreases to 0 as $\varepsilon \rightarrow 0^{+}$such that for any $p \geq 2$,

$$
E|| x_{\varepsilon}(t)-\left.z(t)\right|_{H} ^{p} \leq \psi(\varepsilon) \exp (\xi t), \quad \forall 0 \leq t \leq T .
$$

Next, we turn our attention to a so-called McKean-Vlasov variant of (1.1) given by (1.9) in which $f:[0, T] \times H \times \mathfrak{P}_{\lambda^{2}}(H) \rightarrow H$ and $g:[0, T] \times H \times \mathfrak{P}_{\lambda^{2}}(H) \rightarrow \mathfrak{L}^{2}(K ; H)$ now depend on the probability law $\mu(\cdot)$ of the state process $x(\cdot)$. In addition to (H1)-(H3) and $(\mathrm{H} 7)$, we replace $(\mathrm{H} 4)-(\mathrm{H} 6)$ by the following modified hypothesis:

$(\mathrm{H} 12)$ there exist $\bar{K}:[0, \infty) \times[0, \infty) \times[0, \infty) \rightarrow[0, \infty)$ and $\bar{N}:[0, \infty) \times[0, \infty) \rightarrow[0, \infty)$ satisfying $(\mathrm{H} 4)-(\mathrm{H} 6)$ with $(\mathrm{H} 4)(\mathrm{i})(\mathrm{b})$ and $(\mathrm{H} 4)(\mathrm{ii})(\mathrm{b})$ replaced by

(i) $E\|f(t, x, \mu)\|_{H}^{2}+E\|g(t, x, \mu)\|_{\mathfrak{L}^{2}(K ; H)}^{2} \leq \bar{K}\left(t, E\|x\|_{H}^{2},\|\mu\|_{\lambda^{2}}^{2}\right)$, for all $0 \leq t \leq T$, $\mu \in \mathfrak{P}_{\lambda^{2}}(H)$, and $x \in L^{2}(\Omega ; H)$,

(ii) $E\|f(t, x, \mu)-f(t, y, \nu)\|_{H}^{2}+E\|g(t, x, \mu)-g(t, y, \nu)\|_{\mathfrak{L}^{2}(K ; H)}^{2} \leq \bar{N}\left(t, E\|x-y\|_{H}^{2}\right)+$ $\rho^{2}(\mu, \nu)$, for all $0 \leq t \leq T, \mu, \nu \in \mathfrak{P}_{\lambda^{2}}(H)$, and $x, y \in L^{2}(\Omega ; H)$,

(iii) there exists $\bar{M}_{N}>0$ such that $\bar{N}(t, u) \leq \bar{M}_{N} u$, for all $0 \leq t \leq T$ and $0 \leq u<\infty$.

Remark 3.5. We point out that while the existence portion of the argument for (1.9) can be established using essentially the same argument used to prove Theorem 3.1 without strengthening the assumption on $\bar{N}$, the dependence of $f$ and $g$ on the probability measure $\mu$ creates an additional difficulty when trying to show that $\mu(t)$ is the probability law of $x(t)$. Indeed, it seems that the concavity of $\bar{N}$ in the second variable (which guarantees the existence of positive constants $\alpha_{1}$ and $\alpha_{2}$ such that $\bar{N}(t, u) \leq \alpha_{1}+\alpha_{2} u$, for all $0 \leq t \leq T$ and $0 \leq u<\infty)$ is not quite strong enough. However, taking $\alpha_{1}=0$ (i.e., condition (H12)(ii) becomes a Lipschitz-type condition) is sufficient. Since the nonlinearities involved in McKean-Vlasov equations are often Lipschitz continuous (cf. Example 5.5 in Section 5), the following theorem concerning (1.9) constitutes a reasonable result from the viewpoint of applications; the case of a more general nonlinearity remains an interesting open question.

We have the following analog of Theorem 3.1.

Theorem 3.6. If (H1)-(H3), (H7), and (H12) are satisfied, then (1.9) has a unique mild solution $x \in \mathscr{C}([0, T] ; H)$ for which $\mu(t)$ is the probability distribution of $x(t)$, for all $t \in[0, T]$.

Results analogous to Propositions 3.2 and 3.3 can also be established for (1.9) by making the natural modifications to the hypotheses and proofs.

Finally, in all the previous theorems the operator $A$ in the two definitions of $L$ was independent of $t$. We now briefly comment on the nonautonomous versions of (1.1) and (1.9), where the operator $L(x(t))$ is defined by either (1.2) or (1.3) with $A$ replaced by $\{A(t): 0 \leq t \leq T\}$. In order to proceed in a manner similar to the one currently employed, conditions need to be prescribed under which (i) a resolvent family $\{R(t, s): 0 \leq t \leq s<\infty\}$ is guaranteed to exist, and (ii) it is $\mathfrak{F}_{t}$-adapted. Conditions guaranteeing (i) can be found 
in $[13,20]$, while the approach used in [25] can be modified to establish sufficient conditions that ensure (ii) holds. Once (i) and (ii) hold, each of the results formulated above can be extended to the time-dependent case by making suitable modifications involving the use of the properties of the time-dependent resolvent family (rather than the autonomous one) in the arguments.

\section{Proofs}

Proof of Theorem 3.1. Consider the recursively-defined sequence of successive approximations defined as follows:

$$
\begin{gathered}
x_{0}(t)=R(t) x_{0}, \quad 0 \leq t \leq T \\
x_{n}(t)=R(t) x_{0}+\int_{0}^{t} R(t-s) f\left(s, x_{n-1}(s)\right) d s \\
+\int_{0}^{t} R(t-s) g\left(s, x_{n-1}(s)\right) d W(s), \quad 0 \leq t \leq T, n \geq 1 .
\end{gathered}
$$

Also, consider the initial-value problem

$$
\begin{gathered}
z^{\prime}(t)=C K(t, z(t)), \quad 0 \leq t \leq T, \\
z(0)=z_{0},
\end{gathered}
$$

where $z_{0}>\xi_{1}^{*}+M_{R}\left\|x_{0}\right\|_{L^{2}(H)}^{2}$ and $C=\xi_{2}^{*}$. (Here, $\xi_{1}^{*}=3 M_{R}^{2}\left\|x_{0}\right\|_{L^{2}(H)}^{2}$ and $\xi_{2}^{*}=3 M_{R}^{2}(T+$ $\left.L_{g}\right)$.) Using (H6), we deduce that there exists $0<\bar{T} \leq T$ such that (4.2) has a unique solution $z:[0, \bar{T}] \rightarrow \mathbb{R}$ given by

$$
z(t)=z_{0}+C \int_{0}^{t} K(s, z(s)) d s, \quad \forall 0 \leq t \leq \bar{T}
$$

We will divide the proof of Theorem 3.1 into stages, beginning with the following assertion.

Claim 1. (i) For each $n \geq 1, E\left\|x_{n}(t)\right\|_{H}^{2} \leq z(t)$, for all $0 \leq t \leq \bar{T}$.

(ii) For each $\delta>0$, there exists $0<T^{*} \leq \bar{T}$ (independent of $n$ ) such that

$$
E\left\|x_{n}(t)-R(t) x_{0}\right\|_{H}^{2} \leq \delta
$$

for each $0 \leq t \leq T^{*} \leq \bar{T}$ and for each $n \geq 1$.

Proof. We prove (i) by induction. To begin, for $n=1$, observe that standard computations involving the use of $(\mathrm{H} 4)(\mathrm{i})$ yield

$$
\begin{aligned}
E\left\|x_{1}(t)\right\|_{H}^{2} \leq 3[ & E\left\|R(t) x_{0}\right\|_{H}^{2}+T \int_{0}^{t} E\left\|R(t-s) f\left(s, x_{0}(s)\right)\right\|_{H}^{2} d s \\
& \left.+E\left\|\int_{0}^{t} R(t-s) g\left(s, x_{0}(s)\right) d W(s)\right\|_{H}^{2}\right]
\end{aligned}
$$


10 Stochastic Itó-Volterra integrodifferential equations

$$
\begin{aligned}
& \leq 3 M_{R}^{2}\left\|x_{0}\right\|_{L^{2}(H)}^{2}+3 M_{R}^{2} T \int_{0}^{t} E\left\|f\left(s, x_{0}(s)\right)\right\|_{H}^{2} d s \\
& \quad+3 M_{R}^{2} L_{g} \int_{0}^{t} E\left\|g\left(s, x_{0}(s)\right)\right\|_{\mathfrak{L}^{2}(K ; H)}^{2} d s \\
& \leq 3 M_{R}^{2}\left\|x_{0}\right\|_{L^{2}(H)}^{2}+3 M_{R}^{2}\left[T+L_{g}\right] \int_{0}^{t}\left[E\left\|f\left(s, x_{0}(s)\right)\right\|_{H}^{2}+E\left\|g\left(s, x_{0}(s)\right)\right\|_{\mathfrak{L}^{2}(K ; H)}^{2}\right] d s \\
& \leq \xi_{1}^{*}+\xi_{2}^{*} \int_{0}^{t} K\left(s, E\left\|x_{0}(s)\right\|_{H}^{2}\right) d s \\
& \leq \xi_{1}^{*}+\xi_{2}^{*} \int_{0}^{t} K\left(s, M_{R}^{2}\left\|x_{0}(s)\right\|_{L^{2}(H)}^{2}\right) d s \quad(\text { using monotonicity of } K) \\
& \leq \xi_{1}^{*}+\xi_{2}^{*} \int_{0}^{t} K(s, z(s)) d s \quad\left(\text { by choice of } z_{0}\right) \\
& =z(t)+\left(\xi_{1}^{*}-z_{0}\right) \quad\left(\text { by }(4.3) \text { and choice of } \xi_{2}^{*}\right) \\
& \quad \leq z(t) \quad\left(\text { since } \xi_{1}^{*}<z_{0}\right),
\end{aligned}
$$

for all $0 \leq t \leq \bar{T}$. Now, assume that $E\left\|x_{n}(t)\right\|_{H}^{2} \leq z(t)$, for all $0 \leq t \leq \bar{T}$. Similar computations yield

$$
\begin{aligned}
& E\left\|x_{n+1}(t)\right\|_{H}^{2} \\
& \quad \leq 3 M_{R}^{2}\left\|x_{0}\right\|_{L^{2}(H)}^{2}+3 M_{R}^{2}\left[T+L_{g}\right] \int_{0}^{t}\left[E\left\|f\left(s, x_{n}(s)\right)\right\|_{H}^{2}+E\left\|g\left(s, x_{n}(s)\right)\right\|_{\mathfrak{L}^{2}(K ; H)}^{2}\right] d s \\
& \quad \leq \xi_{1}^{*}+\xi_{2}^{*} \int_{0}^{t} K\left(s, E\left\|x_{n}(s)\right\|_{H}^{2}\right) d s \\
& \left.\quad \leq \xi_{1}^{*}+\xi_{2}^{*} \int_{0}^{t} K(s, z(s)) d s \quad \text { (using the inductive hypothesis and monotonicity of } K\right) \\
& \quad \leq z(t),
\end{aligned}
$$

for all $0 \leq t \leq \bar{T}$. Thus, (i) holds by induction.

Next, in order to prove (ii), let $\delta>0$ be fixed and proceed by induction. For $n=1$, observe that for all $0 \leq t \leq \bar{T}$, we obtain (using (4.3) and the choice of $z_{0}$ )

$$
E\left\|x_{1}(t)-R(t) x_{0}\right\|_{H}^{2} \leq \xi_{2}^{*} \int_{0}^{t} K\left(s,\left\|x_{0}\right\|_{L^{2}(H)}^{2}\right) d s \leq \xi_{2}^{*} \int_{0}^{t} K(s, z(s)) d s .
$$

Also, the continuity of $z$ and $K$ guarantees the existence of $0<T^{*} \leq \bar{T}$ such that

$$
\xi_{2}^{*} \int_{0}^{t} K(s, z(s)) d s \leq \delta, \quad \forall 0 \leq t \leq T^{*} \leq \bar{T},
$$


so that, in conjunction with (4.7), we conclude that

$$
E\left\|x_{1}(t)-R(t) x_{0}\right\|_{H}^{2} \leq \delta, \quad \forall 0 \leq t \leq T^{*} \leq \bar{T} .
$$

Now, assume that $E\left\|x_{n}(t)-R(t) x_{0}\right\|_{H}^{2} \leq \delta$, for all $0 \leq t \leq T^{*} \leq \bar{T}$. Observe that

$$
\begin{aligned}
E\left\|x_{n}(t)-R(t) x_{0}\right\|_{H}^{2} & \leq E\left\|\int_{0}^{t} R(t-s) f\left(s, x_{n}(s)\right) d s+\int_{0}^{t} R(t-s) g\left(s, x_{n}(s)\right) d W(s)\right\|_{H}^{2} \\
& \leq \xi_{2}^{*} \int_{0}^{t} K\left(s, E\left\|x_{n}(s)\right\|_{H}^{2}\right) d s \\
& \leq \xi_{2}^{*} \int_{0}^{t} K(s, z(s)) d s \quad \text { (by Claim 1(i)) } \\
& \leq \delta \quad(\text { by }(4.8)),
\end{aligned}
$$

for all $0 \leq t \leq T^{*} \leq \bar{T}$, as desired. This completes the proof of Claim 1 .

Next, we assert the following.

Claim 2. For all $n, m \geq 1, E\left\|x_{n+m}(t)-x_{n}(t)\right\|_{H}^{2} \leq \xi_{3}^{*} \int_{0}^{t} N(s, 4 \delta) d s$, for all $0 \leq t \leq T^{*} \leq \bar{T}$, where

$$
\xi_{3}^{*}=2 M_{R}^{2}\left[T+L_{g}\right]
$$

Proof. Let $n, m \geq 1$. Routine calculations used in conjunction with the monotonicity of $N$ yield

$$
\begin{aligned}
E\left\|x_{n+m}(t)-x_{n}(t)\right\|_{H}^{2} \leq 2\left[E\left\|\int_{0}^{t} R(t-s)\left[f\left(s, x_{n+m-1}(s)\right)-f\left(s, x_{n-1}(s)\right)\right] d s\right\|_{H}^{2}\right. & \left.\quad+E\left\|\int_{0}^{t} R(t-s)\left[g\left(s, x_{n+m-1}(s)\right)-g\left(s, x_{n-1}(s)\right)\right] d W(s)\right\|_{H}^{2}\right] \\
\leq & \xi_{3}^{*} \int_{0}^{t} N\left(s, E\left\|x_{n+m-1}(s)-x_{n-1}(s)\right\|_{H}^{2}\right) d s \quad(\text { by }(\mathrm{H} 4)(\mathrm{ii})(\mathrm{b})) \\
\leq & \xi_{3}^{*} \int_{0}^{t} N\left(s, 2 E\left[\left\|x_{n+m-1}(s)-R(s) x_{0}\right\|_{H}^{2}+\left\|R(s) x_{0}-x_{n-1}(s)\right\|_{H}^{2}\right]\right) d s \\
\leq & \xi_{3}^{*} \int_{0}^{t} N(s, 4 \delta) d s \quad \text { (by Claim 1(ii)), }
\end{aligned}
$$

for all $0 \leq t \leq T^{*} \leq \bar{T}$. This completes the proof of Claim 2 . 
Next, define the two sequences $\left\{\gamma_{n}(t)\right\}$ and $\left\{\vartheta_{m n}(t)\right\}$ on $\left[0, T^{*}\right]$ as follows:

$$
\begin{gathered}
\gamma_{1}(t)=\xi_{3}^{*} \int_{0}^{t} N(s, 4 \delta) d s, \quad \gamma_{n+1}(t)=\xi_{3}^{*} \int_{0}^{t} N\left(s, \gamma_{n}(s)\right) d s, \quad n \geq 1, \\
\vartheta_{m n}(t)=E\left\|x_{n+m}(t)-x_{n}(t)\right\|_{H}^{2}, \quad n, m \geq 1 .
\end{gathered}
$$

The continuity of $N$ ensures the existence of $0<T^{* *} \leq T^{*}$ such that

$$
\gamma_{1}(t) \leq 4 \delta, \quad \forall 0 \leq t \leq T^{* *}
$$

Claim 3. (i) For all $n \geq 1, \gamma_{n}(t) \leq \gamma_{n-1}(t) \leq \cdots \leq \gamma_{1}(t)$, for all $0 \leq t \leq T^{* *}$.

(ii) For all $m, n \geq 1, \vartheta_{m n}(t) \leq \gamma_{n}(t)$, for all $0 \leq t \leq T^{* *}$.

Proof. We establish (i) using induction on $n$. To begin, we show the string of inequalities holds for $n=2$. To this end, observe that using (4.15) and the monotonicity of $N$ yields

$$
\gamma_{2}(t)=\xi_{3}^{*} \int_{0}^{t} N\left(s, \gamma_{1}(s)\right) d s \leq \xi_{3}^{*} \int_{0}^{t} N(s, 4 \delta) d s=\gamma_{1}(t), \quad \forall 0 \leq t \leq T^{* *}
$$

Now, assume that $\gamma_{n}(t) \leq \gamma_{n-1}(t)$, for all $0 \leq t \leq T^{* *}$, and observe that

$$
\gamma_{n+1}(t)=\xi_{3}^{*} \int_{0}^{t} N\left(s, \gamma_{n}(s)\right) d s \leq \xi_{3}^{*} \int_{0}^{t} N\left(s, \gamma_{n-1}(s)\right) d s=\gamma_{n}(t), \quad \forall 0 \leq t \leq T^{* *} .
$$

This completes the proof of (i). The argumen $\mathrm{t}$ for (ii) is equally as straightforward and will be omitted.

Using Claim 3, we deduce that $\left\{\gamma_{n}(\cdot)\right\}$ is a decreasing sequence in $n$. Moreover, for each given $n \geq 1$, it is easy to see that $\gamma_{n}(t)$ is an increasing function of $t$. Finally, with all of the preliminary work now complete, we can now prove that (1.1) has a mild solution $x$ on $\left[0, T^{* *}\right]$. To this end, define the function $\gamma:\left[0, T^{* *}\right] \rightarrow \mathbb{R}$ by

$$
\gamma(t)=\inf _{n \geq 1} \gamma_{n}(t), \quad \forall 0 \leq t \leq T^{* *} .
$$

Observe that $\gamma$ is nonnegative and continuous, $\gamma(0)=0$, and

$$
\gamma(t) \leq \xi_{3}^{*} \int_{0}^{t} N(s, \gamma(s)) d s, \quad \forall 0 \leq t \leq T^{* *} .
$$

Thus, (H5) implies that $\gamma(t)=0$ on $\left[0, T^{* *}\right]$. Further, observe that Claim 3(ii), together with the monotonicity of $\gamma_{n}(\cdot)$, implies that

$$
\sup _{t \in\left[0, T^{* *}\right]} \vartheta_{m n}(t) \leq \sup _{t \in\left[0, T^{* *}\right]} \gamma_{n}(t) \leq \gamma_{n}\left(T^{* *}\right),
$$

where the right-hand side of (4.20) tends to 0 as $n \rightarrow \infty$. Hence, we deduce from (4.14) that $\left\{x_{n}\right\}$ is a Cauchy sequence in $\mathscr{C}\left(\left[0, T^{* *}\right] ; H\right)$. From completeness, it follows that there 
exists $x \in \mathscr{C}\left(\left[0, T^{* *}\right] ; H\right)$ such that

$$
\left\|x_{n}-x\right\|_{C\left(\left[0, T^{* *}\right] ; H\right)}^{2} \equiv \sup _{t \in\left[0, T^{* *}\right]} E\left\|x_{n}(t)-x(t)\right\|_{H}^{2} \longrightarrow 0 \quad \text { as } n \longrightarrow \infty .
$$

Observe that

$$
\begin{aligned}
& E\left\|\int_{0}^{t} R(t-s)\left[f\left(s, x_{n}(s)\right)-f(s, x(s))\right] d s\right\|_{H}^{2}+E\left\|\int_{0}^{t} R(t-s)\left[g\left(s, x_{n}(s)\right)-g(s, x(s))\right] d W(s)\right\|_{H}^{2} \\
& \quad \leq \xi_{4}^{*} \int_{0}^{t} N\left(s,\left\|x_{n}-x\right\|_{C\left(\left[0, T^{* *}\right] ; H\right)}^{2}\right) d s,
\end{aligned}
$$

where $\xi_{4}^{*}=M_{R}^{2}\left(T+L_{g}\right)$. From the continuity of $N$, (4.21) implies that

$$
N\left(s,\left\|x_{n}-x\right\|_{\mathscr{C}\left(\left[0, T^{* *}\right] ; H\right)}^{2}\right) \rightarrow 0 \quad \text { as } n \rightarrow \infty .
$$

Since $N(t, 0)=0$, for all $0 \leq t \leq T$, we conclude that the left-hand side of (4.22) tends to 0 as $n \rightarrow \infty$. Thus, $x$ is indeed a mild solution of $(1.1)$ on $\left[0, T^{* *}\right]$, as desired. A standard argument can now be employed to prove that the above solution can be extended in finitely many steps to the entire interval $[0, T]$.

Finally, the uniqueness of the mild solution is now easily shown since if $x, y \in \mathscr{C}([0, T]$; $H$ ) are two mild solutions of (1.1), then

$$
\sup _{t \in[0, T]} E\|x(t)-y(t)\|_{H}^{2} \leq 2 M_{R}^{2}\left(T+L_{g}\right) \int_{0}^{t} N\left(s, \sup _{s \in[0, T]} E\|x(s)-y(s)\|_{H}^{2}\right) d s
$$

so that (H5) (with $D=2 M_{R}^{2}\left(T+L_{g}\right)$ ) implies that $\sup _{t \in[0, T]} E\|x(t)-y(t)\|_{H}^{2}=0$. Consequently, $x=y$ in $\mathscr{C}([0, T] ; H)$, thereby showing uniqueness. This completes the proof of Theorem 3.1.

Proof of Theorem 3.4. We proceed by estimating each term of the representation formula (cf. (1.7) and (1.10)) for $E\left\|x_{\varepsilon}(t)-z(t)\right\|_{H}^{p}$ separately. Throughout the proof, $C_{i}$ are positive constants and $\beta_{i}(\varepsilon)$ are positive functions which decrease to 0 as $\varepsilon \rightarrow 0^{+}$. To begin, note that (H9) guarantees the existence of $C_{1}$ and $\beta_{1}(\varepsilon)$ such that for sufficiently small $\varepsilon>0$,

$$
E\left\|R_{\varepsilon}(t) x_{0}-R(t) x_{0}\right\|_{H}^{p} \leq C_{1} \beta_{1}(\varepsilon)
$$

Next, regarding the term $E\left\|\int_{0}^{t}\left[R_{\varepsilon}(t-s) f_{\varepsilon}\left(s, x_{\varepsilon}(s)\right)-R(t-s) f(s, z(s))\right] d s\right\|_{H}^{p}$, the continuity of $f_{\varepsilon}$, together with (H9), ensures the existence of $C_{2}$ and $\beta_{2}(\varepsilon)$ such that for small enough $\varepsilon>0$,

$$
\int_{0}^{t} E\left\|\left[R_{\varepsilon}(t-s)-R(t-s)\right] f(s, z(s))\right\|_{H}^{p} d s \leq C_{2} \beta_{2}(\varepsilon),
$$


14 Stochastic Itó-Volterra integrodifferential equations

for all $0 \leq t \leq T$. Also, observe that Young's inequality and (H10) together yield

$$
\begin{aligned}
& \int_{0}^{t} E\left\|R_{\varepsilon}(t-s)\left[f_{\varepsilon}\left(s, x_{\varepsilon}(s)\right)-f(s, z(s))\right]\right\|_{H}^{p} d s \\
& \quad \leq M_{R}^{p} \int_{0}^{t} E\left\|f_{\varepsilon}\left(s, x_{\varepsilon}(s)\right)-f_{\varepsilon}(s, z(s))+f_{\varepsilon}(s, z(s))-f(s, z(s))\right\|_{H}^{p} d s \\
& \quad \leq 2^{p-1} M_{R}^{p} \int_{0}^{t}\left[M^{p} E\left\|x_{\varepsilon}(s)-z(s)\right\|_{H}^{p}+E\left\|f_{\varepsilon}(s, z(s))-f(s, z(s))\right\|_{H}^{p}\right] d s .
\end{aligned}
$$

Note that (H10) guarantees the existence of $C_{3}$ and $\beta_{3}(\varepsilon)$ such that for small enough $\varepsilon>0$,

$$
E|| f_{\varepsilon}(s, z(s))-f(s, z(s)) \|_{H}^{p} \leq C_{3} \beta_{3}(\varepsilon)
$$

for all $0 \leq t \leq T$, thereby enabling us to conclude from (4.27) that

$$
\begin{aligned}
& \int_{0}^{t} E\left\|R_{\varepsilon}(t-s)\left[f_{\varepsilon}\left(s, x_{\varepsilon}(s)\right)-f(s, z(s))\right]\right\|_{H}^{p} d s \\
& \quad \leq 2^{p-1} M_{R}^{p} M^{p} \int_{0}^{t} E\left\|x_{\varepsilon}(s)-z(s)\right\|_{H}^{p} d s+2^{p-1} M_{R}^{p} T C_{3} \beta_{3}(\varepsilon)
\end{aligned}
$$

for all $0 \leq t \leq T$. Using (4.26) and (4.29) together with the Hölder, Minkowski, and Young inequalities yields

$$
\begin{aligned}
E\left\|\int_{0}^{t}\left[R_{\varepsilon}(t-s) f_{\varepsilon}\left(s, x_{\varepsilon}(s)\right)-R(t-s) f(s, z(s))\right] d s\right\|_{H}^{p} \\
\leq 2^{p-1} E\left[\left(\int_{0}^{t}\left\|R_{\varepsilon}(t-s) f_{\varepsilon}\left(s, x_{\varepsilon}(s)\right)-R_{\varepsilon}(t-s) f(s, z(s))\right\|_{H} d s\right)^{p}\right. \\
\left.+\left(\int_{0}^{t}\left\|R_{\varepsilon}(t-s) f(s, z(s))-R(t-s) f(s, z(s))\right\|_{H} d s\right)^{p}\right] \\
\leq 2^{p-1} T^{p / q}\left[2^{p-1} M_{R}^{p} T C_{3} \beta_{3}(\varepsilon)+2^{p-1} M_{R}^{p} M^{p} \int_{0}^{t} E\left\|x_{\varepsilon}(s)-z(s)\right\|_{H}^{p} d s+C_{2} \beta_{2}(\varepsilon)\right] .
\end{aligned}
$$

Similarly, in order to estimate $E\left\|\int_{0}^{t} R_{\varepsilon}(t-s) g_{\varepsilon}\left(s, x_{\varepsilon}(s)\right) d W(s)\right\|_{H}^{p}$, we note that computations similar to those leading to (4.30), together with Lemma 2.1, yield

$$
\begin{aligned}
& E\left\|\int_{0}^{t} R_{\varepsilon}(t-s) g_{\varepsilon}\left(s, x_{\varepsilon}(s)\right) d W(s)\right\|_{H}^{p} \\
& \quad \leq 2^{p-1} M_{R}^{p}\left[E\left\|\int_{0}^{t}\left(g_{\varepsilon}\left(s, x_{\varepsilon}(s)\right)-g_{\varepsilon}(s, z(s))\right) d W(s)\right\|\left\|_{H}^{p}+E\right\| \int_{0}^{t} g_{\varepsilon}(s, z(s)) d W(s) \|_{H}^{p}\right] \\
& \quad \leq 2^{p-1} M_{R}^{p} L_{g}\left[\int_{0}^{t} M^{p} E\left\|x_{\varepsilon}(s)-z(s)\right\|_{H}^{p} d s+\int_{0}^{t} E\left\|g_{\varepsilon}(s, z(s))\right\|_{\mathfrak{L}^{2}(K ; H)}^{p} d s\right] .
\end{aligned}
$$


(The argument of [16, Proposition 1.9] guarantees the existence of a bound $L_{g}$ (independent of $\varepsilon>0$ ) that applies for all mappings $g_{\varepsilon}$ under consideration.) Also, (H11) guarantees the existence of $C_{4}$ and $\beta_{4}(\varepsilon)$ such that for small enough $\varepsilon>0$,

$$
\int_{0}^{t} E\left\|g_{\varepsilon}(s, z(s))\right\|_{\mathfrak{L}^{2}(K ; H)}^{p} d s \leq C_{4} \beta_{4}(\varepsilon)
$$

for all $0 \leq t \leq T$. Substituting (4.32) in (4.31) yields

$$
E\left\|\int_{0}^{t} R_{\varepsilon}(t-s) g_{\varepsilon}\left(s, x_{\varepsilon}(s)\right) d W(s)\right\|_{H}^{p} \leq 2^{p-1} M_{R}^{p} L_{g}\left[\int_{0}^{t} M^{p} E\left\|x_{\varepsilon}(s)-z(s)\right\|_{H}^{p} d s+C_{4} \beta_{4}(\varepsilon)\right] .
$$

Using (4.33), in conjunction with (4.25)-(4.30), enables us to conclude that for all $\varepsilon>0$ small enough to ensure that (4.25)-(4.33) hold simultaneously, there exists a constant $\eta>0$ (namely, $\eta=2^{p-1} M_{R}^{p} M^{p}\left[2^{p-1} T^{p / q}+L_{g}\right]$ ) such that

$$
E\left\|x_{\varepsilon}(t)-z(t)\right\|_{H}^{p} \leq \sum_{i=1}^{4} C_{i} \beta_{i}(\varepsilon)+\eta \int_{0}^{t} E\left\|x_{\varepsilon}(s)-z(s)\right\|_{H}^{p} d s
$$

An application of Gronwall's lemma in (4.34) subsequently yields

$$
E\left\|x_{\varepsilon}(t)-z(t)\right\|_{H}^{p} \leq \Psi(\varepsilon) \exp (\eta t)
$$

for all $0 \leq t \leq T$, where $\Psi(\varepsilon)=\sum_{i=1}^{4} C_{i} \beta_{i}(\varepsilon)$. This completes the proof of Theorem 3.4.

Proof of Theorem 3.6. Let $\mu \in \mathscr{C}_{\lambda^{2}}(T) \equiv C\left([0, T] ;\left(\mathfrak{P}_{\lambda^{2}}(H), \rho\right)\right)$ be fixed. The existence and uniqueness of a mild solution $x_{\mu}$ on $[0, T]$ of (1.9) can be established as in the proof of Theorem 3.1. We must further show that $\mu$ is, in fact, the probability law of $x_{\mu}$. Toward this end, following the approach used in $[1,19]$, let $\mathfrak{L}\left(x_{\mu}\right)=\left\{\mathfrak{L}\left(x_{\mu}(t)\right): t \in[0, T]\right\}$ denote the probability law of $x_{\mu}$ and define the operator $\Psi$ on $\mathscr{C}_{\lambda^{2}}(T)$ by $\Psi(\mu)=\mathfrak{L}\left(x_{\mu}\right)$. We first prove that $\mathfrak{L}\left(x_{\mu}\right) \in \mathscr{C}_{\lambda^{2}}(T)$; that is, $\Psi$ maps $\mathscr{C}_{\lambda^{2}}(T)$ into itself. Indeed, note that since $x_{\mu} \in \mathscr{C}([0, T] ; H)$, it follows that $\mathfrak{L}\left(x_{\mu}(t)\right) \in \mathfrak{P}_{\lambda^{2}}(H)$, for any $t \in[0, T]$. As such, we only need to show that $t \rightarrow \mathfrak{L}\left(x_{\mu}(t)\right)$ is continuous. To this end, observe that for sufficiently small $|h|>0$, the continuity of $x_{\mu}, \bar{K}$, and $\bar{N}$ implies that

$$
\lim _{h \rightarrow 0} E\left\|x_{\mu}(t+h)-x_{\mu}(t)\right\|_{H}^{2}=0, \quad \forall 0 \leq t \leq T .
$$

Further, for all $t \in[0, T]$ and $\varphi \in \mathfrak{C}_{\rho}(H)$, the definition of the metric $\rho$ (cf. (2.8)) yields

$$
\begin{aligned}
\left|\left\langle\varphi, \mathfrak{L}\left(x_{\mu}(t+h)\right)-\mathfrak{L}\left(x_{\mu}(t)\right)\right\rangle\right| & =\left|E\left[\varphi\left(x_{\mu}(t+h)\right)-\varphi\left(x_{\mu}(t)\right)\right]\right| \\
& \leq\|\varphi\|_{\mathfrak{C}_{\rho}} E\left\|x_{\mu}(t+h)-x_{\mu}(t)\right\|_{H} .
\end{aligned}
$$


Thus, we may conclude that

$$
\lim _{h \rightarrow 0} \rho\left(\mathfrak{L}\left(x_{\mu}(t+h)\right), \mathfrak{L}\left(x_{\mu}(t)\right)\right)=\lim _{h \rightarrow 0} \sup _{\|\varphi\|_{\mathfrak{e}_{\rho} \leq 1}} \int_{H} \varphi(x)\left(\mathfrak{L}\left(x_{\mu}(t+h)\right)-\mathfrak{L}\left(x_{\mu}(t)\right)\right) d x=0,
$$

thereby showing that $\mathfrak{L}\left(x_{\mu}\right) \in \mathscr{C}_{\lambda^{2}}(T)$.

Finally, note that if $x$ is a mild solution of (1.9), then clearly its probability law $\mathfrak{L}(x)=\mu$ is a fixed point of $\Psi$. Conversely, if $\mu$ is a fixed point of $\Psi$, then the variation of parameters representation formula (parametrized by $\mu$ ) defines a solution $x_{\mu}$ which, in turn, has a probability law $\mu$ belonging to the space $\mathscr{C}_{\lambda^{2}}(T)$. Thus, in order to complete the proof it suffices to show that the operator $\Psi$ has a unique fixed point in $\mathscr{C}_{\lambda^{2}}(T)$. To this end, let $\mu$, $\nu$ be any two elements of $\mathscr{C}_{\lambda^{2}}(T)$ and let $x_{\mu}$ and $x_{\nu}$ be the corresponding mild solutions of (1.9). Standard computations employing the use of (H12) yield

$$
E\left\|x_{\mu}(t)-x_{\nu}(t)\right\|_{H}^{2} \leq \bar{M}_{T} \int_{0}^{t}\left[\bar{N}\left(s, E\left\|x_{\mu}(s)-x_{\nu}(s)\right\|_{H}^{2}\right)+\rho^{2}(\mu(s), \nu(s))\right] d s,
$$

where $\bar{M}_{T}=2 M_{R}^{2}\left(T+L_{g}\right)$. Using (H12)(iii), we continue the inequality in (4.39) to obtain

$$
\begin{aligned}
E\left\|x_{\mu}(t)-x_{\nu}(t)\right\|_{H}^{2} & \leq \bar{M}_{T} \int_{0}^{t}\left[\bar{M}_{N} E\left\|x_{\mu}(s)-x_{\nu}(s)\right\|_{H}^{2}+\rho^{2}(\mu(s), v(s))\right] d s \\
& \leq \bar{M}_{T} \int_{0}^{t} \rho^{2}(\mu(s), \nu(s)) d s+\bar{M}_{N} \bar{M}_{T} \int_{0}^{t} E\left\|x_{\mu}(s)-x_{\nu}(s)\right\|_{H}^{2} d s
\end{aligned}
$$

from which it follows by an application of Gronwall's inequality that

$$
\begin{aligned}
E\left\|x_{\mu}(t)-x_{\nu}(t)\right\|_{H}^{2} & \leq \bar{M}_{T}\left(\int_{0}^{t} \rho^{2}(\mu(s), \nu(s)) d s\right) \exp \left(\bar{M}_{N} \bar{M}_{T} t\right) \\
& \leq \bar{M}_{T} \exp \left(\bar{M}_{N} \bar{M}_{T} t\right) t D_{t}^{2}(\mu, \nu) \quad(\text { cf. (2.9)) }
\end{aligned}
$$

for all $0 \leq t \leq T$. Observe that for $0<\bar{T} \leq T$ chosen sufficiently small, there exists a constant $0<C<1$ (independent of $\mu, \nu$ ) for which

$$
\bar{M}_{T} \exp \left(\bar{M}_{N} \bar{M}_{T} t\right) t \leq C, \quad \forall 0 \leq t \leq \bar{T} \leq T,
$$

so that

$$
\bar{M}_{T} \exp \left(\bar{M}_{N} \bar{M}_{T} t\right) t D_{t}^{2}(\mu, \nu) \leq C D \frac{2}{T}(\mu, \nu), \quad \forall 0 \leq t \leq \bar{T} \leq T .
$$

Consequently, from (4.41) and (4.43), we have

$$
E|| x_{\mu}(t)-x_{\nu}(t) \|_{H}^{2} \leq C D \frac{2}{T}(\mu, \nu), \quad \forall 0 \leq t \leq \bar{T}
$$


and therefore

$$
\|\Psi(\mu)-\Psi(\nu)\|_{C_{\lambda^{2}}(\bar{T})}^{2}=D_{\bar{T}}^{2}(\Psi(\mu), \Psi(\nu)) \leq\left\|x_{\mu}-x_{\nu}\right\|_{C_{\lambda^{2}}(\bar{T})}^{2} \leq C D_{\bar{T}}^{2}(\mu, \nu)=C\|\mu-\nu\|_{C_{\lambda^{2}}(\bar{T})}^{2} .
$$

Thus, $\Psi$ is a contraction on $\mathscr{C}_{\lambda^{2}}(\bar{T})$ and therefore, has a unique fixed point. As such, (1.9) has a unique mild solution on $[0, \bar{T}]$ with probability distribution $\mu \in \mathscr{C}_{\lambda^{2}}(\bar{T})$. This procedure can be repeated in order to extend the solution, by continuity, to the entire interval $[0, T]$ in finitely many steps, thereby completing the proof.

\section{Examples}

Example 5.1. Let $D$ be a bounded domain in $\mathbb{R}^{N}$ with smooth boundary $\partial D$. Consider the following initial boundary value problem:

$$
\begin{gathered}
\partial x(t, z)=\left(-\int_{0}^{t} a(t-s) \Delta_{z} x(s, z) d s+F(t, x(t, z))\right) \partial t+G(t, x(t, z)) d \beta(t), \quad \text { a.e. on }(0, T) \times D, \\
x(t, z)=0, \quad \text { a.e. on }(0, T) \times \partial D, \\
x(0, z)=x_{0}(z), \quad \text { a.e. on } D,
\end{gathered}
$$

where $x:[0, T] \times D \rightarrow \mathbb{R}, F:[0, T] \times \mathbb{R} \rightarrow \mathbb{R}, G:[0, T] \times \mathbb{R} \rightarrow \mathfrak{L}^{2}\left(\mathbb{R}^{N}, L^{2}(D)\right), a:[0, T] \rightarrow$ $\mathbb{R}, x_{0}: D \rightarrow \mathbb{R}$, and $\beta$ is a standard $N$-dimensional Brownian motion. We impose the following conditions:

(H13) $a \in L^{1}((0, T)$; $\mathbb{R})$ is an $\mathfrak{F}_{t}$-adapted, positive, nonincreasing, convex kernel;

(H14) $F$ satisfies the Caratheodory conditions (i.e., measurable in $t$ and continuous in $x$ ) and is such that

(i) there exists $\bar{M}_{F}>0$ such that $|F(t, x)| \leq \bar{M}_{F}[1+|x|]$, for all $0 \leq t \leq T$ and $x \in \mathbb{R}$,

(ii) there exists $M_{F}>0$ such that $|F(t, x)-F(t, y)| \leq M_{F}|x-y|$, for all $0 \leq t \leq T$ and $x, y \in \mathbb{R}$;

(H15) $G$ satisfies the Caratheodory conditions and is such that

(i) there exists $\bar{M}_{G}>0$ such that $\|G(t, x)\|_{\mathfrak{L}^{2}\left(\mathbb{R}^{N} ; L^{2}(D)\right)} \leq \bar{M}_{G}[1+|x|]$, for all $0 \leq$ $t \leq T$ and $x \in \mathbb{R}$

(ii) there exists $M_{G}>0$ such that $\|G(t, x)-G(t, y)\|_{\mathfrak{L}^{2}\left(\mathbb{R}^{N} ; L^{2}(D)\right)} \leq M_{G}|x-y|$, for all $0 \leq t \leq T$ and $x, y \in \mathbb{R}$;

(H16) $x_{0}$ is an $\mathfrak{F}_{0}$-measurable random variable independent of $\beta$ with finite second moment.

The following theorem is a stochastic analog of [3, Theorem 6.2].

TheOREM 5.2. If (H13)-(H16) are satisfied, then (5.1) has a unique mild solution $x \in$ $\mathscr{C}\left([0, T] ; L^{2}\left(L^{2}(D)\right)\right)$

Proof. Let $H=L^{2}(D), K=\mathbb{R}^{N}$, and define the operator $A: D(A) \subset H \rightarrow H$ by

$$
A x(t, \cdot)=-\Delta_{z} x(t, \cdot), \quad D(A)=H^{2}(D) \cap H_{0}^{1}(D) .
$$


It is known that $A$ is a positive definite, self-adjoint operator in $H$ (see [3]). Moreover, using the properties of $A$ and (H13), it follows from [25] and [29, page 38] that condition (H3) is satisfied with $M_{R}=1$. Next, $F$ and $G$, respectively, generate functions $f:[0, T] \times$ $H \rightarrow H$ and $g:[0, T] \times H \rightarrow \mathfrak{L}^{2}(K, H)$ by the following identifications:

$$
\begin{array}{ll}
f(t, w)(z)=F(t, w(z)), & \text { a.e. on }(0, T) \times D, \text { for each } w \in H, \\
g(t, w)(z)=G(t, w(z)), & \text { a.e. on }(0, T) \times D, \text { for each } w \in H .
\end{array}
$$

Clearly, $f$ and $g$ satisfy (H4) due to (H14)-(H15), and $x_{0}$ satisfies (H7). Since Lipschitz conditions are imposed in (H14)-(H15), conditions (H5) and (H6) are automatically satisfied (with $N(t, u)=K(t, u)=M u$, where $M=\max \left\{\bar{M}_{F}, \bar{M}_{G}\right\}$ ). We can now rewrite (5.1) in the form (1.1) (with $L$ given by (1.2)) in $H$, and apply Theorem 3.1 to conclude that (5.1) has a unique mild solution $x \in \mathscr{C}\left([0, T] ; L^{2}\left(L^{2}(D)\right)\right)$.

Remark 5.3. Conditions (H14)-(H15) can be weakened by imposing a modified version of (H4) with $N$ and $K$ given by (3.4), for instance. The existence and uniqueness of a mild solution in such case is still guaranteed by Theorem 3.1.

Next, we consider a variant of (5.1) in which the mapping $F$ now depends, in addition, on the probability law of the state process. Precisely, we consider

$$
\begin{aligned}
\partial x(t, z)=( & \left.-\int_{0}^{t} a(t-s) \Delta_{z} x(s, z) d s\right) \partial t+\left(F_{1}(t, z, x(t, z))+\int_{L^{2}(D)} F_{2}(t, z, y) \mu(t, z)(d y)\right) \partial t \\
& +G(t, z, x(t, z)) d \beta(t), \quad \text { a.e. on }(0, T) \times D \\
& x(t, z)=0, \quad \text { a.e. on }(0, T) \times \partial D, \quad x(0, z)=x_{0}(z), \quad \text { a.e. on } D,
\end{aligned}
$$

where $F_{1}:[0, T] \times D \times \mathbb{R} \rightarrow \mathbb{R}, F_{2}:[0, T] \times D \times L^{2}(D) \rightarrow L^{2}(D), G:[0, T] \times D \times \mathbb{R} \rightarrow$ $\mathfrak{L}^{2}\left(\mathbb{R}^{N} ; L^{2}(D)\right)$, and $\mu(t, \cdot) \in \mathfrak{P}_{\lambda^{2}}\left(L^{2}(D)\right)$ is the probability law of $x(t, \cdot)$. We impose the following modified version of hypotheses (H14)-(H15).

(H17) $F_{1}$ satisfies the Caratheodory conditions (i.e., measurable in $(t, z)$ and continuous in the third variable) and is such that

(i) there exists $\bar{M}_{F_{1}}>0$ such that $\left|F_{1}(t, y, z)\right| \leq \bar{M}_{F_{1}}[1+|z|]$, for all $0 \leq t \leq T$, $y \in D, z \in \mathbb{R}$,

(ii) there exists $M_{F_{1}}>0$ such that $\left|F_{1}\left(t, y, z_{1}\right)-F_{1}\left(t, y, z_{2}\right)\right| \leq M_{F_{1}}\left|z_{1}-z_{2}\right|$, for all $0 \leq t \leq T, y \in D, z_{1}, z_{2} \in \mathbb{R}$,

(H18) $F_{2}$ satisfies the Caratheodory conditions and is such that

(i) there exists $\bar{M}_{F_{2}}>0$ such that $\left\|F_{2}(t, y, z)\right\|_{L^{2}(D)} \leq \bar{M}_{F_{2}}\left[1+\|z\|_{L^{2}(D)}\right]$, for all $0 \leq t \leq T, y \in D, z \in L^{2}(D)$,

(ii) $F_{2}(t, y, \cdot): L^{2}(D) \rightarrow L^{2}(D)$ is in $\mathfrak{C}_{\rho}$, for each $0 \leq t \leq T, y \in D$.

(H19) $G$ satisfies the Caratheodory conditions and is such that

(i) there exists $\bar{M}_{G}>0$ such that $\|G(t, y, z)\|_{\mathfrak{L}^{2}\left(\mathbb{R}^{N} ; L^{2}(D)\right)} \leq \bar{M}_{G}[1+|z|]$, for all $0 \leq t \leq T, y \in D, z \in \mathbb{R}$,

(ii) there exists $M_{G}>0$ such that $\left\|G\left(t, y, z_{1}\right)-G\left(t, y, z_{2}\right)\right\|_{\mathfrak{L}^{2}\left(\mathbb{R}^{N} ; L^{2}(D)\right)} \leq M_{G} \mid z_{1}-$ $z_{2} \mid$, for all $0 \leq t \leq T, y \in D, z_{1}, z_{2} \in \mathbb{R}$.

We have the following theorem. 
Theorem 5.4. If (H13) and (H16)-(H19) are satisfied, then (5.4) has a unique mild solution $x \in \mathscr{C}\left([0, T] ; L^{2}\left(L^{2}(D)\right)\right)$ with probability law $\{\mu(t, \cdot): 0 \leq t \leq T\}$.

Proof. Let $H, K$, and $A$ be defined as in the proof of Theorem 5.2. Define the maps $f$ : $[0, T] \times H \times \mathfrak{P}_{\lambda^{2}}(H) \rightarrow H$ and $g:[0, T] \times H \times \mathfrak{P}_{\lambda^{2}}(H) \rightarrow \mathfrak{L}^{2}(K, H)$ by

$$
\begin{gathered}
f(t, x, \mu(t))(z)=F_{1}(t, z, x(t, z))+\int_{L^{2}(D)} F_{2}(t, z, y) \mu(t, z)(d y), \\
g(t, x, \mu(t))(z)=G(t, z, x(t, z)),
\end{gathered}
$$

for all $0 \leq t \leq T, z \in D$, and $x \in H$. We must show that $f$ and $g$ satisfy (H4). To this end, observe that from (H17)(i), we obtain

$$
\begin{aligned}
\left\|F_{1}(t, \cdot, x(t, \cdot))\right\|_{L^{2}(D)} & \leq \bar{M}_{F_{1}}\left[\int_{D}[1+|x(t, z)|]^{2} d z\right]^{1 / 2} \leq 2 \bar{M}_{F_{1}}\left[m(D)+\|x(t, \cdot)\|_{L^{2}(D)}^{2}\right]^{1 / 2} \\
& \leq 2 \bar{M}_{F_{1}}\left[\sqrt{m(D)}+\|x\|_{\mathscr{C}\left(L^{2}(D)\right)}\right] \leq M_{F_{1}}^{*}\left[1+\|x\|_{\mathscr{C}\left(L^{2}(D)\right)}\right]
\end{aligned}
$$

for all $0 \leq t \leq T, x \in \mathscr{C}\left(L^{2}(D)\right)$, where

$$
M_{F_{1}}^{*}= \begin{cases}2 \bar{M}_{F_{1}} \sqrt{m(D)}, & \text { if } m(D)>1, \\ 2 \bar{M}_{F_{1}}, & \text { if } m(D) \leq 1 .\end{cases}
$$

(Here, $m$ denotes the Lebesgue measure in $\mathbb{R}^{n}$.) Also, from (H17)(ii), we obtain

$$
\begin{aligned}
\left\|F_{1}(t, \cdot, x(t, \cdot))-F_{1}(t, \cdot, y(t, \cdot))\right\|_{L^{2}(D)} & \leq M_{F_{1}}\left[\int_{D}|x(t, z)-y(t, z)|^{2} d z\right]^{1 / 2} \\
& \leq M_{F_{1}}\left[\sup _{0 \leq s \leq t}\|x(s, \cdot)-y(s, \cdot)\|_{L^{2}(D)}^{2}\right]^{1 / 2} \\
& =M_{F_{1}}\|x-y\|_{\mathscr{C}\left(L^{2}(D)\right)},
\end{aligned}
$$

for all $0 \leq t \leq T, x, y \in \mathscr{C}\left(L^{2}(D)\right)$. Using (H18)(i), together with the Hölder inequality, yields

$$
\begin{aligned}
\| \int_{L^{2}(D)} F_{2}(t, \cdot, y) \mu(t, \cdot)(d y) & \\
= & {\left[\int_{D}\left[\int_{L^{2}(D)} F_{2}(t, z, y) \mu(t, z)(d y)\right]^{2} d z\right]^{1 / 2} } \\
\leq & {\left[\int_{D} \int_{L^{2}(D)}\left\|F_{2}(t, z, y)\right\|_{L^{2}(D)}^{2} \mu(t, z)(d y) d z\right]^{1 / 2} }
\end{aligned}
$$




$$
\begin{aligned}
& \leq \bar{M}_{F_{2}}\left[\int_{D}\left(\int_{L^{2}(D)}\left(1+\|y\|_{L^{2}(D)}\right)^{2} \mu(t, z)(d y)\right) d z\right]^{1 / 2} \\
& \leq \bar{M}_{F_{2}} \sqrt{m(D)} \sqrt{\|\mu(t)\|_{\lambda^{2}}}(\text { cf. }(2.8)) \\
& \leq \bar{M}_{F_{2}} \sqrt{m(D)}\left(1+\|\mu(t)\|_{\lambda^{2}}\right), \quad \forall 0 \leq t \leq T, \mu(t) \in \mathfrak{P}_{\lambda^{2}}(H) .
\end{aligned}
$$

Also, invoking (H18)(ii) enables us to see that for all $\mu(t), \nu(t) \in \mathfrak{P}_{\lambda^{2}}(H)$,

$$
\begin{aligned}
& \left\|\int_{L^{2}(D)} F_{2}(t, \cdot, y) \mu(t, \cdot)(d y)-\int_{L^{2}(D)} F_{2}(t, \cdot, y) \nu(t, \cdot)(d y)\right\|_{L^{2}(D)} \\
& \quad=\left\|\int_{L^{2}(D)} F_{2}(t, \cdot, y)(\mu(t, \cdot)-v(t, \cdot))(d y)\right\|_{L^{2}(D)} \\
& \leq\|\rho(\mu(t), \nu(t))\|_{L^{2}(D)} \quad(\operatorname{cf} .(2.8)) \\
& =\sqrt{m(D)} \rho(\mu(t), v(t)), \quad \forall 0 \leq t \leq T .
\end{aligned}
$$

Combining (5.7) and (5.10) with (H19)(i), we see that $f$ and $g$ satisfy (H12)(i) with

$$
K(t, u)=M u, \quad \text { where } M=\max \left\{2 \bar{M}_{F_{2}} \sqrt{m(D)}, 2 M_{F_{1}}^{*}, \bar{M}_{G}\right\}
$$

Similarly, combining (5.9) and (5.11) with (H19)(ii), we see that $f$ and $g$ satisfy (H12)(ii) with

$$
N(t, u)=\bar{M} u, \quad \text { where } \bar{M}=\max \left\{M_{F_{1}}, \sqrt{m(D)}, M_{G}\right\}
$$

Thus, we can invoke Theorem 3.6 to conclude that (5.4) has a unique mild solution $x \in$ $\mathscr{C}\left([0, T] ; L^{2}\left(L^{2}(D)\right)\right)$ with probability law $\{\mu(t, \cdot): 0 \leq t \leq T\}$.

Example 5.5. The following is a stochastic version of the classical initial-boundary value problem governing heat flow through materials with memory, which has been studied in [21]:

$$
\begin{gathered}
q(t, z)=-\left(x_{z}(t, z)+\int_{0}^{t} a(t-s) x_{z}(s, z) d s\right), \quad 0 \leq t \leq T, 0 \leq z \leq L, \\
\partial x(t, z)=\left(-\frac{\partial}{\partial z} q(t, z)+F(t, z)\right) \partial t+G(t, z) \partial W(t), \quad 0 \leq t \leq T, 0 \leq z \leq L, \\
x(0, z)=x_{0}(z), \quad 0 \leq z \leq L,
\end{gathered}
$$

where $F:[0, T] \times[0, L] \rightarrow \mathbb{R}, G:[0, T] \times[0, L] \rightarrow \mathfrak{L}^{2}\left(\left(L^{2}(0, L)\right)^{2}\right), x_{0}:[0, L] \rightarrow \mathbb{R}$, and $W$ is a standard $L^{2}(0, L)$-valued Wiener process. Here, we assume that $a, F$, and $G$ satisfy (H13)-(H16), appropriately adapted to the current setting. 
We identify $H=K=L^{2}(0, L)$ and define $A: D(A) \subset H \rightarrow H$ by

$$
A x(t, \cdot)=-\frac{\partial^{2}}{\partial^{2} z} x(t, \cdot), \quad D(A)=H^{2}(0, L) \cap H_{0}^{1}(0, L) .
$$

It is known that $(A, a)$ satisfies (H3), and that $F$ and $G$ define functions $f$ and $g$ as in Example 5.1. As such, (5.14) can be written in the abstract form (1.1) with $L$ given by (1.3). Arguing as before, one can invoke Theorem 3.1 to prove that (5.14) has a unique mild solution $x \in \mathscr{C}\left([0, T] ; L^{2}\left(L^{2}(0, L)\right)\right)$.

Remark 5.6. (i) If $F$ in (5.14) is replaced by a mapping as defined in (5.5), then the resulting initial-value problem constitutes a McKean-Vlasov variant of (5.14). Arguing as in Theorem 5.4, one can invoke Theorem 3.6 to conclude the existence and uniqueness of a mild solution in such case.

(ii) A concrete example of (1.1) (with $L$ given by (1.3)) that arises in the study of viscoelasticity is discussed in $[22,23]$. A stochastic version of this example in the spirit of those discussed in this section can be established in a similar manner, assuming that conditions comparable to those in [25] are imposed to ensure the existence of an $\mathfrak{F}_{t^{-}}$ adapted resolvent family.

\section{Acknowledgment}

The authors would like to express their sincere gratitude to the anonymous reviewers for their careful reading of the manuscript, as well as their comments that led to a considerable improvement of the original manuscript.

\section{References}

[1] N. U. Ahmed and X. Ding, A semilinear McKean-Vlasov stochastic evolution equation in Hilbert space, Stochastic Processes and Their Applications 60 (1995), no. 1, 65-85.

[2] S. Aizicovici and K. B. Hannsgen, Local existence for abstract semilinear Volterra integrodifferential equations, Journal of Integral Equations and Applications 5 (1993), no. 3, 299-313.

[3] S. Aizicovici and M. A. McKibben, Semilinear Volterra integrodifferential equations with nonlocal initial conditions, Abstract and Applied Analysis 4 (1999), no. 2, 127-139.

[4] A. T. Bharucha-Reid, Random Integral Equations, Mathematics in Science and Engineering, vol. 96, Academic Press, New York, 1972.

[5] R. W. Carr and K. B. Hannsgen, A nonhomogeneous integro-differential equation in Hilbert space, SIAM Journal on Mathematical Analysis 10 (1979), no. 5, 961-984.

[6] _ Resolvent formulas for a Volterra equation in Hilbert space, SIAM Journal on Mathematical Analysis 13 (1982), no. 3, 459-483.

[7] Gh. Constantin, The uniqueness of solutions of perturbed backward stochastic differential equations, Journal of Mathematical Analysis and Applications 300 (2004), no. 1, 12-16.

[8] G. Da Prato and M. Iannelli, Linear integro-differential equations in Banach spaces, Rendiconti del Seminario Matematico dell'Università di Padova 62 (1980), 207-219.

[9] G. Da Prato and J. Zabczyk, Stochastic Equations in Infinite Dimensions, Encyclopedia of Mathematics and Its Applications, vol. 44, Cambridge University Press, Cambridge, 1992.

[10] D. A. Dawson, Critical dynamics and fluctuations for a mean-field model of cooperative behavior, Journal of Statistical Physics 31 (1983), no. 1, 29-85.

[11] D. A. Dawson and J. Gärtner, Large deviations from the McKean-Vlasov limit for weakly interacting diffusions, Stochastics 20 (1987), no. 4, 247-308. 
[12] Y. El Boukfaoui and M. Erraoui, Remarks on the existence and approximation for semilinear stochastic differential equations in Hilbert spaces, Stochastic Analysis and Applications 20 (2002), no. 3, 495-518.

[13] R. C. Grimmer, Resolvent operators for integral equations in a Banach space, Transactions of the American Mathematical Society 273 (1982), no. 1, 333-349.

[14] K. B. Hannsgen and R. L. Wheeler, Behavior of the solution of a Volterra equation as a parameter tends to infinity, Journal of Integral Equations 7 (1984), no. 3, 229-237.

[15] Y. Hu and N. Lerner, On the existence and uniqueness of solutions to stochastic equations in infinite dimension with integral-Lipschitz coefficients, Journal of Mathematics of Kyoto University $\mathbf{4 2}$ (2002), no. 3, 579-598.

[16] A. Ichikawa, Stability of semilinear stochastic evolution equations, Journal of Mathematical Analysis and Applications 90 (1982), no. 1, 12-44.

[17] D. Kannan, Random integrodifferential equations, Probabilistic Analysis and Related Topics, Vol. 1 (A. R. Bharucha-Reid, ed.), Academic Press, New York, 1978, pp. 87-167.

[18] D. Kannan and A. T. Bharucha-Reid, On a stochastic integro-differential evolution equation of Volterra type, Journal of Integral Equations 10 (1985), no. 1-3, suppl., 351-379.

[19] D. N. Keck and M. A. McKibben, Abstract stochastic integrodifferential delay equations, Journal of Applied Mathematics and Stochastic Analysis 2005 (2005), no. 3, 275-305.

[20] J. A. León and D. Nualart, Stochastic evolution equations with random generators, The Annals of Probability 26 (1998), no. 1, 149-186.

[21] Y. P. Lin and J. H. Liu, Semilinear integrodifferential equations with nonlocal Cauchy problem, Nonlinear Analysis 26 (1996), no. 5, 1023-1033.

[22] J. H. Liu, Integrodifferential equations with non-autonomous operators, Dynamic Systems and Applications 7 (1998), no. 3, 427-439.

[23] J. H. Liu and K. Ezzinbi, Non-autonomous integrodifferential equations with non-local conditions, Journal of Integral Equations and Applications 15 (2003), no. 1, 79-93.

[24] Yu. S. Mīshura, Existence of solutions of abstract Volterra equations in a Banach space and its subsets, Ukraïns' kiĭ Matematichniǔ Zhurnal 52 (2000), no. 5, 648-657, English translation in Ukrainian Mathematical Journal 52 (2000), no. 5, 741-753 (2001).

[25] _ Abstract Volterra equations with stochastic kernels, Theory of Probability and Mathematical Statistics (2002), no. 64, 139-151.

[26] Yu. S. Mīshura and Yu. V. Tomīlov, The method of successive approximations for abstract Volterra equations in a Banach space, Ukraïns' kiŭ Matematichnir Zhurnal 51 (1999), no. 3, 376-382, English translation in Ukrainian Mathematical Journal 51 (1999), no. 3, 419-426.

[27] M. Nagasawa and H. Tanaka, Diffusion with interactions and collisions between coloured particles and the propagation of chaos, Probability Theory and Related Fields 74 (1987), no. 2, 161-198.

[28] J. Prüss, Positivity and regularity of hyperbolic Volterra equations in Banach spaces, Mathematische Annalen 279 (1987), no. 2, 317-344.

[29] __ Evolutionary Integral Equations and Applications, Monographs in Mathematics, vol. 87, Birkhäuser, Basel, 1993.

David N. Keck: Department of Mathematics, Ohio University, Athens, OH 45701, USA

E-mail address: dkeck@bing.math.ohiou.edu

Mark A. McKibben: Department of Mathematics and Computer Science, Goucher College,

Baltimore, MD 21204, USA

E-mail address: mmckibben@goucher.edu 


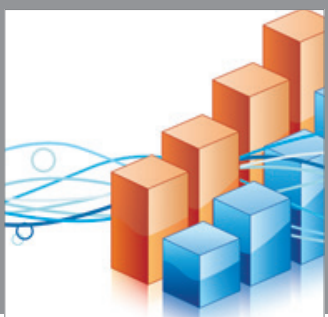

Advances in

Operations Research

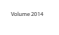

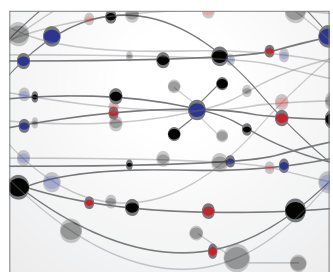

\section{The Scientific} World Journal
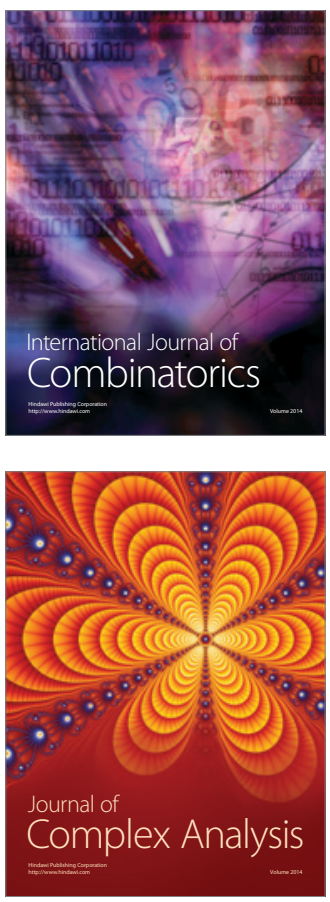

International Journal of

Mathematics and

Mathematical

Sciences
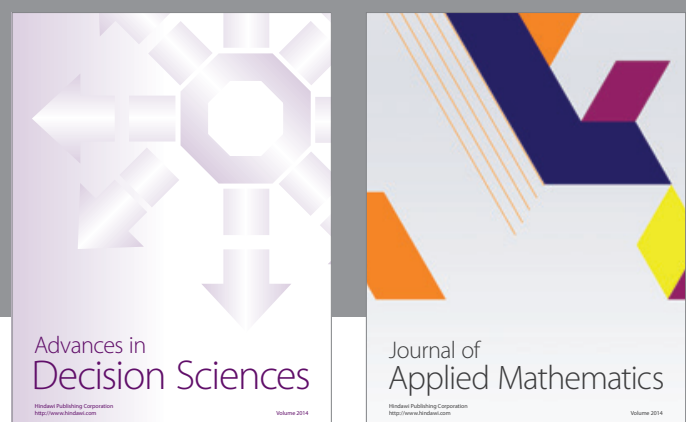

Journal of

Applied Mathematics
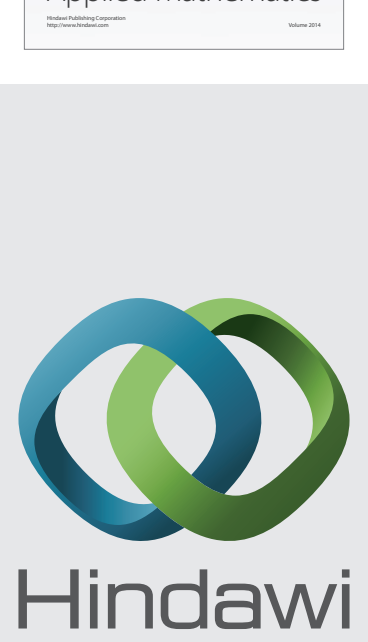

Submit your manuscripts at http://www.hindawi.com
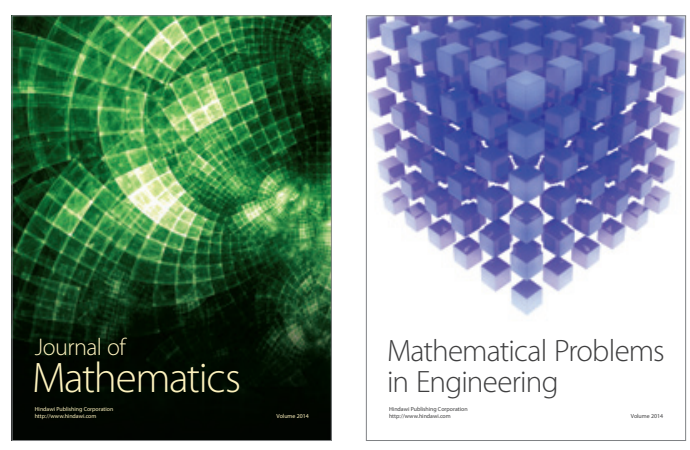

Mathematical Problems in Engineering
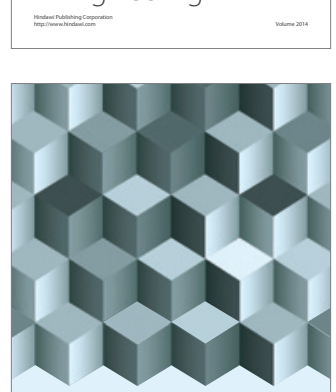

Journal of

Function Spaces
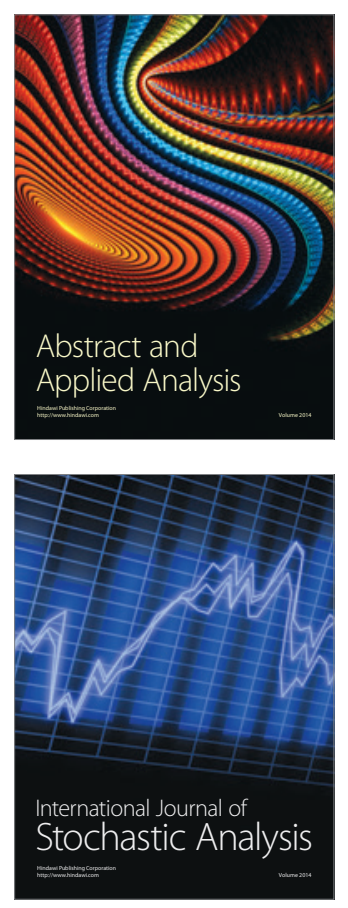

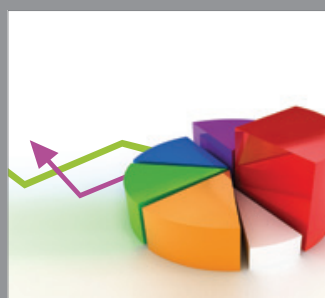

ournal of

Probability and Statistics

Promensencen
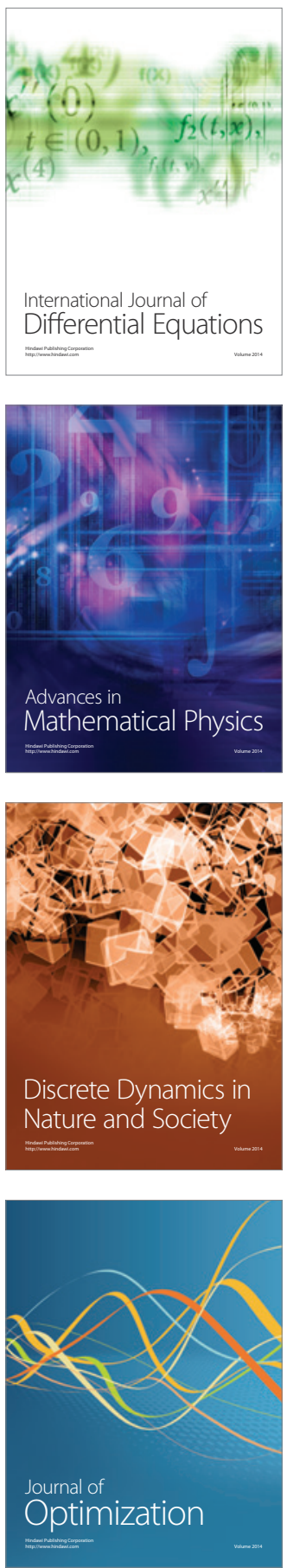